\title{
Identification of the risk factors in perioperative respiratory adverse events in children under general anesthesia and the development of a predictive model
}

\author{
Shoujun Tao ${ }^{1}$, Tao Zhang ${ }^{2}$, Kai Wang ${ }^{3}$, Fanghua $\mathrm{Xie}^{4}$, Lifeng $\mathrm{Ni}^{4}$, Zhong $\mathrm{Mei}^{4}$, Shaobo Song ${ }^{4}$ \\ ${ }^{1}$ Department of Anesthesiology, Affiliated Hangzhou First People's Hospital, Zhejiang University School of Medicine, Hangzhou, China; \\ ${ }^{2}$ Department of Anesthesiology, Taizhou Hospital of Zhejiang Province Affiliated to Wenzhou Medical University, Taizhou, China; ${ }^{3}$ Department of \\ Urology, Affiliated Hangzhou First People's Hospital, Zhejiang University School of Medicine, Hangzhou, China; ${ }^{4}$ Department of Anesthesiology, \\ Affiliated Xiaoshan Hospital, Hangzhou Normal University, Hangzhou, China \\ Contributions: (I) Conception and design: S Tao, T Zhang, S Song; (II) Administrative support: K Wang, F Xie; (III) Provision of study materials \\ or patients: L Ni, Z Mei, S Song; (IV) Collection and assembly of data: All authors; (V) Data analysis and interpretation: S Tao, T Zhang, S Song; \\ (VI) Manuscript writing: All authors; (VII) Final approval of manuscript: All authors. \\ Correspondence to: Shaobo Song. Department of Anesthesiology, Affiliated Xiaoshan Hospital, Hangzhou Normal University, 728 Yucai North Road, \\ Hangzhou 311201, China. Email: songshaobo@zju.edu.cn.
}

Background: This study explored the risk factors of perioperative respiratory adverse events in children under 12 years old undergoing general anesthesia surgery. A prediction model was constructed according to the related risk factors to provide a basis for timely clinical intervention and decision-making.

Methods: Children under 12 years old who underwent general anesthesia in our hospital between January 2016 and December 2020 were included in this study. The clinical data, including age, gender, weight, American Society of Anesthesiologists (ASA) grade classification, operation season, preoperative hospital stay, anesthesia time, and postoperative pain score, were collated. Continuous variables were converted to categorical variables. Logistic regression analysis was used to screen independent risk factors and a nomogram was constructed to predict the probability of adverse events. Fitting curves and receiver operating characteristic (ROC) curves were utilized to verify the model.

Results: Logistic regression analyses demonstrated that age [odds ratio $(\mathrm{OR})=1.32$, 95\% confidence interval (CI): 1.08 to 1.49 ], body weight ( $\mathrm{OR}=1.49,95 \% \mathrm{CI}: 1.21$ to 1.84$)$, anesthesia time (OR $=1.61$, 95\% CI: 1.32 to 1.78 ), and surgery season ( $\mathrm{OR}=1.12,95 \% \mathrm{CI}: 1.07$ to 1.39 ) were independent risk factors for respiratory adverse events in children undergoing general anesthesia $(\mathrm{P}<0.05)$. The risk of respiratoryrelated adverse events increased in children with grade II ASA classification compared to children with grade I ASA classification $(\mathrm{P}<0.05)$. Similarly, the risk of respiratory adverse events increased in children with level III pain scores compared to children with level I pain scores $(\mathrm{P}<0.05)$. The calibration curve showed that the predicted curve was consistent with the actual curve. The area under the ROC curve (AUC) was 0.707, indicating that model showed great predictive ability.

Conclusions: Age, weight, anesthesia time, operation season, ASA grade, and pain score were identified as independent risk factors for respiratory adverse events in children undergoing general anesthesia. Using the above risk factors, a nomogram was established to predict the risk of respiratory system-related adverse events. The predicted results were highly consistent with the actual risk, and the false positive rate was within a reasonable range.

Keywords: Pediatric anesthesia; adverse events; logistics regression analysis; nomogram

Submitted May 20, 2021. Accepted for publication Jun 29, 2021.

doi: $10.21037 / \mathrm{tp}-21-257$

View this article at: https://dx.doi.org/10.21037/tp-21-257 


\section{Introduction}

Due to advances in surgical and medical-support technology, surgery has become a widely accepted treatment option for children with diseases (1-3). However, a child's under-developed physical and anatomical structure is significantly different from that of the full grown adult. Therefore, pediatric surgeries cannot follow the same standards or routines as adult operations. In fact, different stages of a child's development will require variation in the drug regimens, anesthesia methods, and operation methods. Furthermore, a child's tissues and organs are more vulnerable to damage and their immune function is weaker compared to an adult. However, their tissue regeneration and repair are more efficient compared to an adult. In surgical operations, children are more sensitive to narcotic drugs, and are less tolerant to anesthesia and surgical procedures compared to adults. They have a higher incidence of postoperative complications and adverse events $(4,5)$. For children undergoing general anesthesia, the incidence of adverse events may be associated with age and the professional skill of the anesthesiologists. Studies have shown that the older the child, the lower the incidence of adverse events, especially in children under 12 years of old. Experienced pediatric anesthesiologists can also reduce the risk of adverse events (6-9). Timely treatment of adverse events, such as airway obstruction caused by mucosal edema and tongue drop and perioperative hypothermia, is essential for the preservation of life. In addition, accurate risk assessment of general anesthesia in the perioperative period can allow for early intervention and risk aversion, thereby improving postoperative treatment and clinical outcomes. Given this clinical demand, the current study explored the risk factors of perioperative respiratory adverse events in children under 12 years old undergoing general anesthesia. A prediction model was constructed according to the relevant risk factors to provide a basis for timely clinical intervention and decision-making. We present the following article in accordance with the TRIPOD reporting checklist (available at https://dx.doi.org/10.21037/tp-21-257).

\section{Methods}

\section{Patients}

Children under 12 years old who underwent general anesthesia in our hospital from January 2016 to December 2020 were included in the study. Children were included in the study if they were (I) less than 12 years old; (II) showed
American Society of Anesthesiologists (ASA) grade IIII classification; and (III) underwent elective surgery and general anesthesia with endotracheal intubation. Children were excluded if they: (I) underwent throat or airway-related surgery; (II) presented with cardiovascular, mental or nervous system diseases, or other primary severe diseases; (III) were allergic to inhaled anesthetics; and (IV) participated in other clinical trials. The legal guardians of all participants were fully aware of the research and agreed to participate. All procedures performed in this study involving human participants were in accordance with the Declaration of Helsinki (as revised in 2013). The study was approved by the Ethics Committee of the Affiliated Xiaoshan Hospital, Hangzhou Normal University (No. 2021-066) and informed consent was taken from all the patients.

\section{Anesthesia method}

The patient's electrocardiograph and other vital signs including body temperature, pulse, respiratory rate, blood pressure, and oxygenation index, were continuously monitored. For the induction of anesthesia, propofol with remifentanil was injected intravenously, and sevoflurane was administered by inhalation during the operation. Muscle relaxants were administered if necessary. The attending anesthesiologist was responsible for the anesthesia-related procedure and the subsequent follow-up.

\section{Observation index}

The age, gender, weight, ASA grade, operation season, duration of preoperative hospitalization, anesthesia time, and postoperative pain score were recorded. Vital signs (including body temperature, pulse, respiratory rate, and blood pressure) were measured before the operation, during the operation, during recovery, and 24 hours after the surgery. Pain score was assessed 24 hours after the operation.

\section{Conversion of variables}

Patients were divided according to age, namely $\leq 1$ and $>1$ year old. Body weight was classified as normal or abnormal (with abnormal defined as $20 \%$ above or below the normal pediatric weight). The operation season was split into spring to summer and autumn to winter. The length of hospital stay before the operation was classified into two groups, namely $\leq 3$ and $>3$ days. The anesthesia time was defined as 
induction of anesthesia to the completion of recovery, which was classified as $\leq 1$ or $>1$ hour. At 24 hours post-operation, a pain score of 0-3 was considered grade I, a score of 4-6 was grade II, and a score of $7-10$ was grade III.

\section{Adverse events}

The incidence of postoperative adverse events was monitored. Low oxygen saturation was defined as oxygen saturation less than $95 \%$ at rest. Pyrexia was defined as a temperature greater than $38^{\circ} \mathrm{C}$. Ventilation dysfunction due to the tongue falling back, breath-holding, laryngospasm, or bronchospasm was assessed. Other clinical symptoms, including cough and phlegm, were also monitored.

\section{Statistical analysis}

The $\mathrm{R}$ language and related $\mathrm{R}$ packages were applied for statistical analyses. The count data was expressed as a percentage. The independent sample $t$-tests were used for data comparison between groups and chi-square tests were used for count data. Logistic regression analysis was used to screened independent risk factors which were then used to constructed a nomogram to predict the probability of adverse events. The fitting curves and receiver operating characteristic (ROC) curves were applied to verify the model.

\section{Results}

\section{General results}

A total of 476 children were enrolled in this study, with a median age of 7 years, ranging from 11 days to 12 years old. There were 141 cases $(29.62 \%) \leq 1$ year old and 335 cases $(70.38 \%)>1$ year old. The median body weight was $31.4 \mathrm{~kg}$, ranging from 2.8 to $61.7 \mathrm{~kg}$. A total of 75 children (15.76\%) presented with abnormal body weight, and 401 children (84.24\%) had normal body weight. There were 301 males (63.24\%) and 175 females (36.76\%). A total of 254 cases were classified as ASA grade I (53.36\%), 143 cases were grade II $(30.04 \%)$, and 79 cases were grade III (16.60\%). There were 221 operations $(46.43 \%)$ during spring and summer, and $53.57 \%$ of operations occurred during autumn and winter. A total of 297 cases (62.39\%) were hospitalized less than 3 days before the operation and 179 cases (37.61\%) were hospitalized more than 3 days before the operation. The anesthesia time was $\leq 1$ hour in 189 cases $(39.71 \%$ ) and $>1$ hour in 287 cases $(60.29 \%)$. Grade I pain scores were recorded for 173 patients (36.34\%), 201 cases $(42.23 \%)$ recorded grade II pain scores, and 102 cases $(21.43 \%)$ recorded grade III pain scores 24 hours after the operation. Within 48 hours of anesthesia induction, 263 children $(55.25 \%)$ experienced respiratory-related adverse events, and no adverse events were detected in 213 patients $(44.75 \%)$.

\section{Analysis of independent risk factors}

Clinical factors were converted to categorical variables, including age, gender, weight, ASA grade, pain score, duration of anesthesia, duration of preoperative hospital stay, and season of operation time. Logistic regression analysis indicated that age [odds ratio $(\mathrm{OR})=1.32,95 \%$ confidence interval (CI): 1.08 to 1.49], weight $(\mathrm{OR}=1.49$, 95\% CI: 1.21 to 1.84$)$, duration of anesthesia $(\mathrm{OR}=1.61$, $95 \% \mathrm{CI}: 1.32$ to 1.78$)$, and season of surgery $(\mathrm{OR}=1.12$, $95 \%$ CI: 1.07 to 1.39 ) were independent risk factors for respiratory adverse events in children undergoing general anesthesia surgery $(\mathrm{P}<0.05)$. Children with grade II $(\mathrm{OR}=1.04,95 \% \mathrm{CI}: 0.79$ to 1.16$)$ and grade III $(\mathrm{OR}=1.13$, 95\% CI: 0.81 to 1.25 ) ASA classification had an increased risk of respiratory-related adverse events compared to grade I ASA patients $(\mathrm{P}<0.05)$. Furthermore, patients with grade III pain scores (OR $=1.31,95 \%$ CI: 1.07 to 1.44 ) had an increased risk of developing respiratory-related adverse events compared to patients with grade I pain scores $(\mathrm{P}<0.05 ;$ Table 1).

\section{Establishing a nomogram to prediction the risk of adverse events}

Logistic regression models were used to develop nomograms for predicting the risk of respiratory adverse events in children undergoing general anesthesia surgery. Seven variables, including age, weight, ASA grade, pain score, duration of anesthesia, and surgery season, were finally included in this study. Each variable corresponded to a different score standard. After adding up the scores, the risk of suffering an adverse event could be calculated directly. As the score approaches 1 , the risk of adverse events increases. Conversely, as the score approaches 0 , the risk of adverse events decreases (Figure 1).

\section{Establishment of a calibration curve}

Based on the nomogram prediction model, the risk 
of possible respiratory adverse events in children was calculated and represented as a calibration curve. The predicted results were highly consistent with the actual

Table 1 Analysis of the independent risk factors for respiratoryrelated adverse events

\begin{tabular}{|c|c|c|}
\hline Variables & OR $(95 \% \mathrm{Cl})$ & $P$ value \\
\hline Age & $1.32(1.08,1.49)$ & $<0.05$ \\
\hline Gender & $0.68(0.11,0.98)$ & $>0.05$ \\
\hline Body weight & $1.49(1.21,1.84)$ & $<0.05$ \\
\hline \multicolumn{3}{|l|}{ ASA class } \\
\hline I & Reference & \\
\hline II & $1.04(0.79,1.16)$ & $<0.05$ \\
\hline III & $1.13(0.81,1.25)$ & $<0.05$ \\
\hline \multicolumn{3}{|l|}{ Plain score } \\
\hline 1 & Reference & \\
\hline II & $1.57(1.51,1.89)$ & $>0.05$ \\
\hline III & $1.31(1.07,1.44)$ & $<0.05$ \\
\hline Anesthesia duration & $1.61(1.32,1.78)$ & $<0.05$ \\
\hline $\begin{array}{l}\text { Preoperative } \\
\text { hospitalization time }\end{array}$ & $0.37(0.19,0.81)$ & $>0.05$ \\
\hline Season of surgery & $1.12(1.07,1.39)$ & $<0.05$ \\
\hline
\end{tabular}

OR, odds ratio; $\mathrm{Cl}$, confidence interval; ASA, American Society of Anesthesiologists. situation (Figure 2).

\section{Validation of ROC curve}

The ROC curve was constructed according to the falsepositive rate and actual positive rate. The area under the ROC curve (AUC) was 0.707 , indicating that the model had a good predictive ability (Figure 3).

\section{Discussion}

Since children are still developing, their anatomy and physiological functions are significantly different from those of adults. Thus, the technical manipulation of general anesthesia is more challenging and its associated adverse events are more prevalent in children than in adults $(10,11)$. Nearly two decades of research have shown that anesthetic drugs can modify cognitive structure and function, particularly in children. Furthermore, the respiratory-related adverse events in children can be severe. Children tend to be more sensitive to medications, less tolerant of surgical procedures, and have higher rates of postoperative complications and adverse events compared to adults. Studies have shown that the age of the child and the experience of the anesthesiologists are correlated to the incidence of adverse events caused by general anesthesia (12-15). Therefore, children who are older tend to have fewer adverse events, and effective pediatric anesthesia can

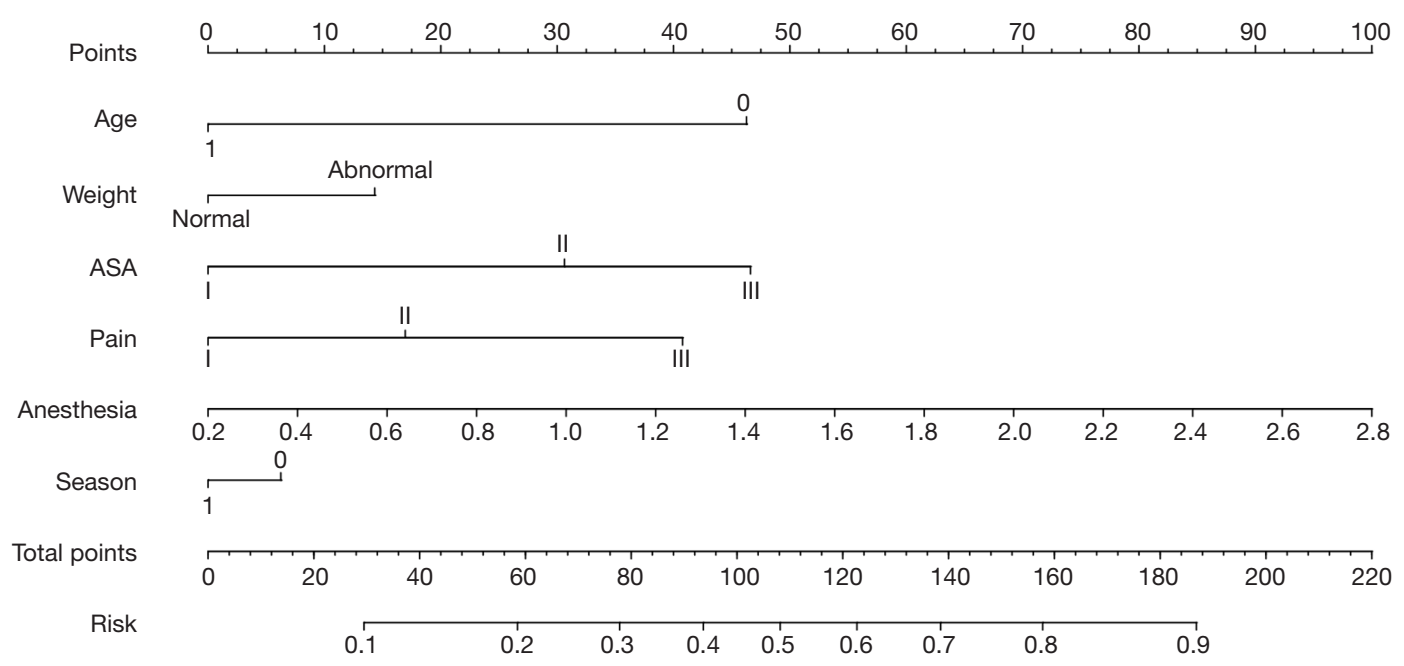

Figure 1 Nomogram to predict the risk of respiratory adverse events in children undergoing general anesthesia surgery. ASA, American Society of Anesthesiologists. 


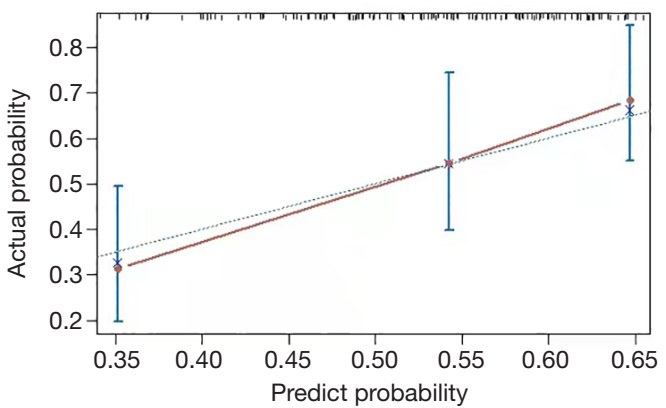

Figure 2 The calibration curve of the nomogram for the risk of respiratory adverse events in children undergoing surgery under general anesthesia. The dotted line represents the actual occurrence risk. The red line represents the predicted occurrence risk. The abscissa represents the predicted risk and the ordinate represents the actual risk.

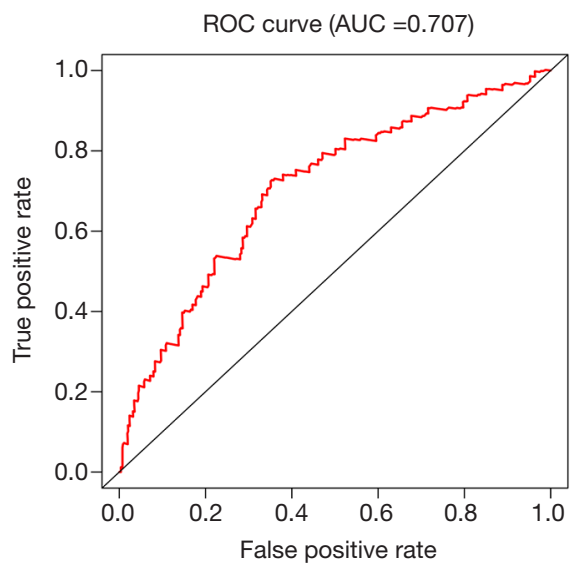

Figure 3 The ROC curve. The AUC was 0.707. The abscissa represents the false positive rate and the ordinate represents the true positive rate. ROC, receiver operating characteristic; AUC, area under the ROC curve.

reduce the risk of adverse events in children. Adverse events such as respiratory obstruction caused by mucosal edema and post-lingual fall, and perioperative hypothermia require immediate intervention to avoid fatal outcomes (16-18). Thus, accurate risk assessment during the perioperative period is crucial to allow for early intervention and risk avoidance measures. This can considerably improve the results of postoperative treatment, resulting in better clinical outcomes.

This study determined that age, weight, anesthesia duration, and surgical season were independent risk factors for respiratory adverse events in children undergoing surgery under general anesthesia $(\mathrm{P}<0.05)$. Children with grade II and III ASA classification showed an increased the risk of developing respiratory-related adverse events compared to grade I patients $(\mathrm{P}<0.05)$. Similarly, patients with grade III pain scores had an increased risk of developing respiratory-related adverse events compared to patients with grade I pain scores $(\mathrm{P}<0.05)$. The calibration curve and ROC curve indicated that the nomogram built using the above risk factors had good predictive value for respiratory-related adverse events. The predicted results were consistent with the actual risk. It is worth noting that previous studies have shown that, in addition to our findings, perioperative upper respiratory tract infection is an independent risk factor for perioperative respiratory adverse events in children. That is, the perioperative upper respiratory tract infection leads to an increase in the incidence of respiratory adverse events, including the asymptomatic period of upper respiratory tract infection (4). However, this study believes that upper respiratory tract infections will be treated before elective surgery, so it was not studied as a variable.

In summary, age, weight, duration of anesthesia, surgery season, ASA class, and pain score were independent risk factors for respiratory adverse events in children undergoing surgery under general anesthesia. This study developed a nomogram that could predicted the risk of respiratoryrelated adverse events with good predictive ability. Moreover, the predicted results were highly consistent with the actual risk, and the false-positive rate was within a reasonable range.

\section{Acknowledgments}

Funding: None.

\section{Footnote}

Reporting Checklist: The authors have completed the TRIPOD reporting checklist. Available at https://dx.doi. org/10.21037/tp-21-257

Data Sharing Statement: Available at https://dx.doi. org/10.21037/tp-21-257

Conflicts of Interest: All authors have completed the ICMJE uniform disclosure form (available at https://dx.doi. org/10.21037/tp-21-257). The authors have no conflicts of interest to declare. 
Ethical Statement: The authors are accountable for all aspects of the work in ensuring that questions related to the accuracy or integrity of any part of the work are appropriately investigated and resolved. All procedures performed in this study involving human participants were in accordance with the Declaration of Helsinki (as revised in 2013). The study was approved by the Ethics Committee of the Affiliated Xiaoshan Hospital, Hangzhou Normal University (No. 2021-066) and informed consent was taken from all the patients.

Open Access Statement: This is an Open Access article distributed in accordance with the Creative Commons Attribution-NonCommercial-NoDerivs 4.0 International License (CC BY-NC-ND 4.0), which permits the noncommercial replication and distribution of the article with the strict proviso that no changes or edits are made and the original work is properly cited (including links to both the formal publication through the relevant DOI and the license). See: https://creativecommons.org/licenses/by-nc-nd/4.0/.

\section{References}

1. Basak D. Pediatric surgery: is it growing? J Indian Assoc Pediatr Surg 2019;24:87-8.

2. Harting MT, Wheeler A, Ponsky T, et al. Telemedicine in pediatric surgery. J Pediatr Surg 2019;54:587-94.

3. Dubousset J. Past, present, and future in pediatric spinal surgery. Ann Transl Med 2020;8:36.

4. Chowdhary SK. Pediatric surgery. Indian J Pediatr 2008;75:923.

5. Azarow KS, Cusick RA. Pediatric surgery. Surg Clin North Am 2012;92:xvii-xix.

6. Baetzel AE, Holman A, Dobija N, et al. Racial Disparities in Pediatric Anesthesia. Anesthesiol Clin 2020;38:327-39.

Cite this article as: Tao S, Zhang T, Wang K, Xie F, Ni L, Mei Z, Song S. Identification of the risk factors in perioperative respiratory adverse events in children under general anesthesia and the development of a predictive model. Transl Pediatr 2021;10(7):1877-1882. doi: 10.21037/tp-21-257
7. Adler AC, Matisoff AJ, DiNardo JA, et al. Point-ofcare ultrasound in pediatric anesthesia: perioperative considerations. Curr Opin Anaesthesiol 2020;33:343-53.

8. Becke K. Pediatric anesthesia: back to the future! Curr Opin Anaesthesiol 2015;28:300-1.

9. Obara S, Kuratani N. Training in pediatric anesthesia in Japan: how should we come along? J Anesth 2020. [Epub ahead of print]. doi: 10.1007/s00540-020-02859-8.

10. Andersson H, Schmitz A, Frykholm P. Preoperative fasting guidelines in pediatric anesthesia: are we ready for a change? Curr Opin Anaesthesiol 2018;31:342-8.

11. Tesoro S, Marchesini L, De Robertis E. Pediatric anesthesia. Transl Med UniSa 2019;20:1-3.

12. Chidambaran V, Costandi A, D'Mello A. Propofol: a review of its role in pediatric anesthesia and sedation. CNS Drugs 2015;29:543-63. Erratum in: CNS Drugs 2018;32:873.

13. Moore AD, Anghelescu DL. Emergence delirium in pediatric anesthesia. Paediatr Drugs 2017;19:11-20. Erratum in: Paediatr Drugs 2017;19:267.

14. Wu JP. Pediatric anesthesia concerns and management for orthopedic procedures. Pediatr Clin North Am 2020;67:71-84.

15. Hansen TG. Editorial: Pediatric anesthesia research: quo vadis? Curr Opin Anaesthesiol 2019;32:325-6.

16. Kuratani N, Kanmura Y. Pediatric anesthesia: current status and future directions. J Anesth 2016;30:185-6.

17. Lai LL, See MH, Rampal S, et al. Significant factors influencing inadvertent hypothermia in pediatric anesthesia. J Clin Monit Comput 2019;33:1105-12.

18. Barton K, Nickerson JP, Higgins T, et al. Pediatric anesthesia and neurotoxicity: what the radiologist needs to know. Pediatr Radiol 2018;48:31-6.

(English Language Editor: J. Teoh) 